\title{
POWER INTEGRAL BASES \\ IN A FAMILY OF SEXTIC FIELDS WITH QUADRATIC SUBFIELDS
}

\author{
István GaÁL — LÁsZló ReMete
}

\begin{abstract}
Let $M=\mathbb{Q}(i \sqrt{d})$ be any imaginary quadratic field with a positive square-free $d$. Consider the polynomial

$$
f(x)=x^{3}-a x^{2}-(a+3) x-1
$$

with a parameter $a \in \mathbb{Z}$. Let $K=M(\alpha)$, where $\alpha$ is a root of $f$. This is an infinite parametric family of sextic fields depending on two parameters, $a$ and $d$. Applying relative Thue's equations we determine the relative power integral bases of these sextic fields over their quadratic subfields. Using these results we also determine generators of (absolute) power integral bases of the sextic fields.
\end{abstract}

\section{Introduction}

Monogenity is a classical topic of algebraic number theory c.f. [4], [14]. Let $M \subset K$ be algebraic number fields with $[K: M]=n$, denote by $\mathbb{Z}_{M}$ and $\mathbb{Z}_{K}$ the rings of integers of $M$ and $K$, respectively. An order $\mathcal{O}$ of $\mathbb{Z}_{K}$ is monogene over $M$ if $\mathcal{O}=\mathbb{Z}_{M}[\vartheta]$ with some $\vartheta \in \mathcal{O}$. In this case $\left(1, \vartheta, \ldots, \vartheta^{n-1}\right)$ is a power integral basis of $\mathcal{O}$ over $M$. If $\mathcal{O}=\mathbb{Z}_{K}$, then we call $K$ monogene over $M$. In the absolute case if $M=\mathbb{Q}$, then we call $K$ monogene and $\left(1, \vartheta, \ldots, \vartheta^{n-1}\right)$ a power integral basis of $K$.

In the relative case $\alpha, \beta \in \mathcal{O}$ are called equivalent if $\alpha=a+\varepsilon \beta$ with an $a \in \mathbb{Z}_{M}$ and with a unit $\varepsilon \in \mathbb{Z}_{M}$. In the absolute case (if $M=\mathbb{Q}$ ) this simplifies to $\alpha=a \pm \beta$ with an $a \in \mathbb{Z}$. If $\alpha$ is equivalent to $\beta$, then $\alpha$ generates a power integral basis if and only if $\beta$ does: we determine generators of power integral bases only up to equivalence.

(C) 2015 Mathematical Institute, Slovak Academy of Sciences.

2010 Mathematics Subject Classification: Primary 11R04; Secondary 11Y50.

Keyw ords: sextic fields, relative cubic extension, power integral basis, relative power integral basis.

Research supported in part by K115479 from the Hungarian National Foundation for Scientific Research. 
There are several algorithms for deciding monogenity and determining generators of power integral bases. These procedures heavily depend on the degree and other properties of number fields, see [4].

We also considered monogenity in infinite parametric families of number fields in the absolute and relative cases, as well, see I. Gaál and G. Lettl [5], I. Gaál and T. Szabó [8, 9].

Recently I. Gaál, L. Remete and T. Szabó [7] studied the relation of monogenity and relative monogenity which was already applied in a family of octic fields by I. G a ál and L. R e m et e [6]. In the present paper we utilize similar tools to describe power integral bases in a well known infinite parametric family of sextic fields.

\section{A parametric family of sextic fields}

Throughout this paper $d$ will be a positive square-free integer. Set $M=\mathbb{Q}(i \sqrt{d})$ with ring of integers $\mathbb{Z}_{M}$. Let

$\omega=i \sqrt{d} \quad$ if $-d \equiv 2,3 \quad(\bmod 4) \quad$ and $\quad \omega=(1+i \sqrt{d}) / 2 \quad$ if $-d \equiv 1 \quad(\bmod 4)$.

Consider the polynomial

$$
f(x)=x^{3}-a x^{2}-(a+3) x-1,
$$

with a parameter $a \in \mathbb{Z}$. These well-known polynomials correspond to the simplest cubic fields of D. Sh a nks [15] studied by several authors. The discriminant of the polynomial is

$$
D(f)=\left(a^{2}+3 a+9\right)^{2} .
$$

Let $\alpha$ be a root of $f$. Remark that $\alpha$ depends on the parameter $a$. Our purpose is to determine generators of power integral bases in the sextic fields $K=M(\alpha)$, more exactly in the order

$$
\mathcal{O}=\mathbb{Z}\left[1, \alpha, \alpha^{2}, \omega, \omega \alpha, \omega \alpha^{2}\right]
$$

of $K$. The field $K$ is just the composite of $M=\mathbb{Q}(i \sqrt{d})$ and $L=\mathbb{Q}(\alpha)$. As it is well known (see [14]) if $\left\{1, \alpha, \alpha^{2}\right\}$ is an integral basis in $L$ and the discriminants of $L$ and $M$ are coprime, then we have $\mathcal{O}=\mathbb{Z}_{K}$. According to [16], if $a^{2}+3 a+9$ is square-free, then $\left\{1, \alpha, \alpha^{2}\right\}$ is indeed an integral basis in $L$. 


\section{Simplest cubics over imaginary quadratic fields}

We shall use a result of C. He u berger [10] on the solutions of the parametric family of the relative Thue equations corresponding to the simplest cubic fields. Consider the equation

$$
Y_{1}^{3}-a Y_{1} Y_{2}^{2}-(a+3) Y_{1} Y_{2}^{2}-Y_{2}^{3}=\varepsilon \quad\left(Y_{1}, Y_{2} \in \mathbb{Z}_{M}\right),
$$

where $\varepsilon$ is a unit in $M$. This parametric family of the relative Thue equation was studied by C. Heuberger, A. Pethö and R. F. Tichy 12 who gave the solutions for large parameters and by C. Heuberger [10], who gave the solutions for all parameters (even for quadratic integer parameters). His result was extended by P. Kirs chen hofer, C. M. L a mpl and J. Thusw ald ner [13] involving also a wider class of rights hand sides. Here we use a special case of H e u b er g e r's result (see also [1]):

LEMma 1. Let $\omega_{3}=(1+i \sqrt{3}) / 2$. Up to sign equation (44) has the following solutions independent of " $a$ ":

$$
\begin{aligned}
& \left(Y_{1}, Y_{2}\right)= \\
& (1,0),(0,1),(1,-1),(i,-i),(i, 0),(0, i), \\
& \left(\omega_{3},-\omega_{3}\right),\left(0, \omega_{3}\right),\left(\omega_{3}, 0\right),\left(1-\omega_{3}, 0\right),\left(0,1-\omega_{3}\right),\left(1-\omega_{3},-1+\omega_{3}\right)
\end{aligned}
$$

and the following solutions depending on " $a$ ":

$$
\begin{aligned}
& \left(a, Y_{1}, Y_{2}\right)= \\
& (-3,9,-2),(-3,7,-9),(-3,2,7),(-1,3,-1),(-1,2,-3),(-1,1,2),(0,9,-4), \\
& (0,5,-9),(0,4,5),(0,2,-1),(0,1,1),(0,1,-2),(1,9,-5),(1,5,4),(1,4,-9), \\
& (1,1,1),(1,1,-2),(2,3,-2),(2,2,1),(2,1,-3),(4,9,-7),(4,7,2),(4,2,-9), \\
& (1,2,-1) .
\end{aligned}
$$

\section{Results}

Using the results of I. G a ál, L. R e m e te and T. S z a bó [7] we show that the generators of relative power integral bases of $\mathcal{O}$ over $M$ can be obtained from the solutions of the above relative Thue equation. As a consequence of Lemma 1 we have the following proposition which will be proved in Section 6 .

Proposition 2. Up to equivalence all generators of relative power integral bases of $\mathcal{O}$ over $M$ are of the form $X_{1} \alpha+X_{2} \alpha^{2}$ where $X_{1}=Y_{1}-a Y_{2}, X_{2}=Y_{2}$, and $\left(Y_{1}, Y_{2}\right)$ is a solution of equation (4). 


\section{ISTVÁN GAÁL — LÁSZLÓ REMETE}

Our main result is on the generators of (absolute) power integral bases of $\mathcal{O}$ :

TheOREM 3. The order $\mathcal{O}$ is only monogene for $d=1$ for the following values of "a", otherwise $\mathcal{O}$ is not monogene. For $d=1$ these values of " $a$ " together with the coordinates (listed up to sign) $y \in \mathbb{Z}, X_{1}, X_{2} \in \mathbb{Z}_{M}$ of the generators

$$
y \omega+X_{1} \alpha+X_{2} \alpha^{2}
$$

of power integral bases of $\mathcal{O}$ are given by

$$
\begin{aligned}
& \left(a, y, X_{1}, X_{2}\right)= \\
& (-3,1, i+a i,-i),(-2,1, i+a i,-i),(-1,1, i+a i,-i),(0,1, i+a i,-i), \\
& (-3,2, i+a i,-i),(-2,2, i+a i,-i),(-1,2, i+a i,-i),(0,2, i+a i,-i), \\
& (-3,1, i, 0),(-2,1, i, 0),(-1,1, i, 0),(0,1, i, 0), \\
& (-3,0, i, 0),(-2,0, i, 0),(-1,0, i, 0),(0,0, i, 0,), \\
& (-2,0,-i a, i),(-1,-1,-i a, i),(0,-2,-i a, i),(-3,1,-i a, i), \\
& (0,-3,-i a, i),(-3,0,-i a, i),(-1,-2,-i a, i),(-2,-1,-i a, i) .
\end{aligned}
$$

\section{Absolute and relative monogenity}

The discriminant of the basis (3) of $\mathcal{O}$ is

$$
D_{\mathcal{O}}=D(f)^{2} \cdot D_{M}^{3} .
$$

Using the method of [7] we shall first determine generators of relative power integral bases of $\mathcal{O}$ over $M$ and then generators of (absolute) power integral bases of $\mathcal{O}$.

Denote by $\omega^{(1)}$ and $\omega^{(2)}$ the conjugates of $\omega \in M$. Let $\alpha^{(i)}(i=1,2,3)$ be the roots of $f$. Any $\vartheta \in \mathcal{O}$ can be written in the form

$$
\vartheta=x_{0}+x_{1} \alpha+x_{2} \alpha^{2}+y_{0} \omega+y_{1} \omega \alpha+y_{2} \omega \alpha^{2}
$$

with $x_{0}, x_{1}, x_{2}, y_{0}, y_{1}, y_{2} \in \mathbb{Z}$. Set

$$
\vartheta^{(i, j)}=x_{0}+x_{1} \alpha^{(j)}+x_{2}\left(\alpha^{(j)}\right)^{2}+y_{0} \omega^{(i)}+y_{1} \omega^{(i)} \alpha^{(j)}+y_{2} \omega^{(i)}\left(\alpha^{(j)}\right)^{2}
$$

for $1 \leq i \leq 2,1 \leq j \leq 3$. Let

$$
I_{\mathcal{O} / M}(\vartheta)=\left(\mathcal{O}^{+}: \mathbb{Z}_{M}[\vartheta]^{+}\right)=\frac{1}{|(D(f))|} \cdot \prod_{i=1}^{2} \prod_{1 \leq j_{1}<j_{2} \leq 3}\left|\vartheta^{\left(i, j_{1}\right)}-\vartheta^{\left(i, j_{2}\right)}\right|
$$

and

$$
J(\vartheta)=\left(\mathbb{Z}_{M}[\vartheta]^{+}: \mathbb{Z}[\vartheta]^{+}\right)=\frac{1}{\left(\sqrt{\left|D_{M}\right|}\right)^{3}} \cdot \prod_{j_{1}=1}^{3} \prod_{j_{2}=1}^{3}\left|\vartheta^{\left(1, j_{1}\right)}-\vartheta^{\left(2, j_{2}\right)}\right| .
$$


In our case Proposition 1 of I. Gaál, L. Remete and T. Szabó 7] gets the following from:

Lemma 4. We have

$$
I(\vartheta)=I_{\mathcal{O} / M}(\vartheta) \cdot J(\vartheta),
$$

where the first factor is the relative index of $\vartheta$ over $M$.

Obviously, $\vartheta$ generates a power integral basis in $\mathcal{O}$ if and only if its index (10) is equal to 1 , that is both the relative index (8) and the factor (9) has to be equal to 1.

\section{Calculating generators of relative power integral bases}

Pro of of Proposition 2 First we calculate those $\vartheta \in \mathcal{O}$ for which the relative index (8) is 1 . These elements generate a relative power integral basis of $\mathcal{O}$ over $M$. As we have shown in I. G a ál [3], calculating generators of relative power integral bases in cubic relative extensions leads to the cubic relative Thue equations.

Using the notation (7) for $i=1,2, j_{1}<j_{2}$ we have

$$
\vartheta^{\left(i, j_{1}\right)}-\vartheta^{\left(i, j_{2}\right)}=\left(\alpha^{\left(j_{1}\right)}-\alpha^{\left(j_{2}\right)}\right)\left(X_{1}^{(i)}+\left(\alpha^{\left(j_{1}\right)}+\alpha^{\left(j_{2}\right)}\right) X_{2}^{(i)}\right)
$$

with quadratic integers

$$
X_{1}=x_{1}+\omega y_{1}, \quad X_{2}=x_{2}+\omega y_{2} \quad \text { in } M .
$$

We have

$$
\alpha^{(1)}+\alpha^{(2)}+\alpha^{(3)}=a, \quad \text { that is, if } j=\{1,2,3\} \backslash\left\{j_{1}, j_{2}\right\},
$$

then

with

$$
\begin{aligned}
X_{1}^{(i)}+\left(\alpha^{\left(j_{1}\right)}+\alpha^{\left(j_{2}\right)}\right) X_{2}^{(i)} & =X_{1}^{(i)}+\left(a-\alpha^{(j)}\right) X_{2}^{(i)} \\
& =\left(X_{1}^{(i)}+a X_{2}^{(i)}\right)-\alpha^{(j)} X_{2}^{(i)} \\
& =Y_{1}^{(i)}-\alpha^{(j)} Y_{2}^{(i)}
\end{aligned}
$$

We have

$$
Y_{1}=X_{1}+a X_{2}, \quad Y_{2}=X_{2} .
$$

$$
\begin{aligned}
\prod_{i=1}^{2} \prod_{1 \leq j_{1}<j_{2} \leq 3}\left|\vartheta^{\left(i, j_{1}\right)}-\vartheta^{\left(i, j_{2}\right)}\right| & = \\
& \left(\prod_{i=1}^{2} \prod_{1 \leq j_{1}<j_{2} \leq 3}\left|\alpha^{\left(j_{1}\right)}-\alpha^{\left(j_{2}\right)}\right|\right) \cdot\left|N_{M / Q}\left(N_{K / M}\left(Y_{1}-\alpha Y_{2}\right)\right)\right| .
\end{aligned}
$$


Therefore by (8) the equation

$$
I_{\mathcal{O} / M}(\vartheta)=1
$$

is just equivalent to

$$
N_{M / Q}\left(N_{K / M}\left(Y_{1}-\alpha Y_{2}\right)\right)= \pm 1 .
$$

This is just the relative Thue equation (4) corresponding to the polynomial $f$.

If $\left(Y_{1}, Y_{2}\right)$ is a solution of the relative Thue equation, then by (11) we calculate

$$
X_{1}=Y_{1}-a Y_{2}, \quad X_{2}=Y_{2} .
$$

Using this transformation, Lemma 1 implies Proposition 2.

\section{Calculating generators of power integral bases}

Proof of Theorem 3. Generators of power integral bases are determined only up to sign, and translation by elements in $\mathbb{Z}$. In view of Lemma 4 , given $\left(X_{1}, X_{2}\right)$ we have to determine $A=x_{0}+\omega y_{0}$ and the unit $\varepsilon$ in $M$ so that for

$$
\vartheta=x_{0}+\omega y_{0}+\varepsilon\left(X_{1} \alpha+X_{2} \alpha^{2}\right)
$$

we have

$$
J(\vartheta)=1 .
$$

The index is translation invariant, indeed $J(\vartheta)$ does not depend on $x_{0}$. Therefore we only have to determine $y_{0}$. This seems to be easy, however several solutions $\left(X_{1}, X_{2}\right)$ are independent from the parameter $a$ and from $d$. This means that in several cases the equation (14) has three variables and degree 9.

I. Consider first the solutions $\left(Y_{1}, Y_{2}\right)=(1,-1),(1,0),(0,1)$ which are independent from $d$ and $a$. Let

$$
X_{1}=Y_{1}-a Y_{2}, \quad X_{2}=Y_{2} .
$$

I.1. Let $-d \equiv 2,3(\bmod 4),-d \neq-1$. Taking any of $\left(Y_{1}, Y_{2}\right)=(1,-1),(1,0),(0,1)$ we have the same arguments. $J(\vartheta)$ is divisible by $y_{0}^{3}$, therefore $y_{0}= \pm 1$. The remaining factor $J_{1}$ of $J(\vartheta)$ is quadratic in $y_{0}$, hence we may substitute $y_{0}=1$ to get $J_{2}$. It is easily seen that $J_{2} \equiv 1(\bmod 4)$ both for even and odd values of $a$. Therefore $J_{2}-1=0$ must be satisfied for a generator of a power integral basis. Set $K=a^{2}+3 a+9$, then

$$
J_{2}-1=K^{2}(4 d+1)+K\left(23 d^{2}\right)+\left(64 d^{3}\right) .
$$

This equation is quadratic in $K$ with discriminant $-64 d^{3}+4 d+1$ which is negative for any integer $d>0$ which excludes the existence of a power integral basis. 
I.2. Let $-d \equiv 1(\bmod 4),-d \neq-3$. Taking any of $\left(Y_{1}, Y_{2}\right)=(1,-1),(1,0),(0,1)$ we have the following arguments. $J(\vartheta)$ is divisible by $y_{0}^{3}$, therefore $y_{0}= \pm 1$. The remaining factor $J_{1}$ of $J(\vartheta)$ is quadratic in $y_{0}$, hence we may substitute $y_{0}=1$ to get $J_{2}$. Then $J_{2} \pm 1=0$ must hold for a solution. Set

$$
\begin{gathered}
K=a^{2}+3 a+9, \\
J_{2}=(d+1) K^{2}+2 d^{2} K+d^{3} .
\end{gathered}
$$

Equation $J_{2}-1=0$ has discriminant $-d^{3}+d+1$, equation $J_{2}+1=0$ has discriminant $-d^{3}-d-1$ in $K$. Both of them are negative for any $d>0$, $-d \equiv 1(\bmod 4)$.

II. Consider the Gaussian integers $\left(Y_{1}, Y_{2}\right)=(i,-i),(i, 0),(0, i)$. In this case $d=1$. For each pair $\left(Y_{1}, Y_{2}\right)$ we calculate $X_{1}, X_{2}$ and take $\varepsilon=1$ and $\varepsilon=i$ in (13) (these are all units up to sign). Then $J(\vartheta)$ depends only on $a$ and $y_{0}$. In each case $J(\vartheta)$ has two factors, say $G_{1}, G_{2}$, one of being a complete square. We solved the polynomial equations $G_{1}= \pm 1, G_{2}= \pm 1$ by using resolvents and found the solutions listed in Theorem 3

II.1. Similarly, we tested $\left(Y_{1}, Y_{2}\right)=(1,-1),(1,0),(0,1)$ with $d=1$ and $\varepsilon=1, i$ which did not give any solutions.

III. Consider the Eulerian integers $\left(Y_{1}, Y_{2}\right)=\left(\omega_{3},-\omega_{3}\right),\left(0, \omega_{3}\right),\left(\omega_{3}, 0\right),\left(1-\omega_{3}, 0\right)$, $\left(0,1-\omega_{3}\right),\left(1-\omega_{3},-1+\omega_{3}\right)$. In this case $d=3$. For each pair $\left(Y_{1}, Y_{2}\right)$ we calculate $X_{1}, X_{2}$ and take $\varepsilon=1, \omega_{3}, \omega_{3}^{2}$ in (13) (these are all units up to sign). Then $J(\vartheta)$ depends only on $a$ and $y_{0}$. In each case $J(\vartheta)$ has two factors, say $G_{1}, G_{2}$. Solving the polynomial equations $G_{1}= \pm 1, G_{2}= \pm 1$ by using resolvents we did not find any integer solutions.

III.1. Similarly, we tested $\left(Y_{1}, Y_{2}\right)=(1,-1),(1,0),(0,1)$ with $d=3$ and $\varepsilon=1, \omega_{3}, \omega_{3}^{2}$ which did not give any solutions.

IV. Consider the solutions $\left(a, Y_{1}, Y_{2}\right)$ given in Lemma 1 . The parameter $a$ is given, we calculate $X_{1}=Y_{1}-a Y_{2}, X_{2}=Y_{2}$. Using suitable units $\varepsilon$ we calculate $J(\vartheta)$ which always had two factors $G_{1}, G_{2}$. Solving the polynomial equations $G_{1}= \pm 1, G_{2}= \pm 1$ by using resolvents we did not find any integer solutions.

This proves Theorem 3 ,

Computational remarks. All our computations were performed in Maple [1] and was executed on an average laptop. The calculations took some seconds only. 


\section{ISTVÁN GAÁL — LÁSZLÓ REMETE}

\section{REFERENCES}

[1] CHAR, B.W.-GEDDES, K.O.-GONNET, G.H.-MONAGAN, M.B.-WATT, S.M. (EDS.): MAPLE, Reference Manual, Watcom Publications, Waterloo, Canada, 1988.

[2] GAÁL, I.: Power integral bases in composits of number fields, Canad. Math. Bull. 41 (1998), 158-161.

[3] GAÁL, I.: Power integral bases in cubic relative extensions, Experiment. Math. 10 (2001), 133-139.

[4] GAÁL, I.: Diophantine equations and power integral bases. Boston, Birkhäuser, 2002.

[5] GAÁL, I.-LETTL, G.: A parametric family of quintic Thue equations II., Monatsh. Math. 131 (2000), 29-35.

[6] GAÁL, I.-REMETE, L.: Non-monogenity in a family of octic fields, Rocky Mountain J. Math. (to appear).

[7] GAÁL, I.-REMETE, L.-SZABÓ, T.: Calculating power integral bases by using relative power integral bases, Funct. Approx. Comment. Math. (to appear).

[8] GAÁL, I.-SZABÓ, T.: Power integral bases in parametric families of biquadratic fields, JP J. Algebra Number Theory Appl. 21 (2012), 105-114.

[9] GAÁL, I.-SZABÓ, T.: Relative power integral bases in infinite families of quartic extensions of quadratic field, JP J. Algebra Number Theory Appl. 29 (2013), 31-43.

[10] HEUBERGER, C.: All solutions to Thomas family of Thue equations over imaginary quadratic number fields, J. Symbolic Comput. 41 (2006), 980-998.

[11] HEUBERGER, C.: All solutions to Thomas family of Thue equations over imaginary quadratic number fields. Online resources, 2006, http://www.opt.math.tu-graz.ac.at/ cheub/publications/thuerel-hyper-online.html

[12] HEUBERGER, C.-PETHÖ, A.-TICHY, R. F.: Thomas family of Thue equations over imaginary quadratic fields, J. Symbolic Comput. 34 (2002), 437-449.

[13] KIRSCHENHOFER, P.-LAMPL, C. M.-THUSWALDNER, J.: On a parameterized family of relative Thue equations Publ. Math. (Debrecen) 71 (2007) 101-139.

[14] NARKIEWICZ, W.: Elementary and Analytic Theory of Algebraic Numbers (2nd ed.). Springer, Berlin, 1974.

[15] SHANKS, D.: The simplest cubic fields, Math. Comput. 28 (1974), 1137-1152.

[16] WASHington, L. C.: Class numbers of the simplest cubic fields, Math. Comp. 48 (1987), 371-384.

E-mail: gaal.istvan@unideb.hu remetel $42 @$ gmail.com 